triple therapy as a first line. If a second attempt at eradication fails, children have oesophagogastroduodenoscopy (OGD) on a dedicated gastroenterology/microbiology $\mathrm{H}$. pylori culture list. Subsequent antibiotic therapy is then based on antibiotic sensitivity.

Aims Our primary objective was to study the benefit of $\mathrm{H}$. pylori eradication therapy based on culture and sensitivity in our population. Our secondary objective was to describe the sensitivities of the enumerated $\mathrm{H}$. pylori.

Subjects and Methods We retrospectively included all paediatric patients who had undergone OGD for $\mathrm{H}$. pylori culture in Royal Hospital for Children, Glasgow, between 2014 and 2020. We collected data from patient electronic records. $\mathrm{H}$. pylori colonisation was based on the presence/absence of the organism on histopathology. Eradication was assessed by either H. pylori stool antigen or subsequent gastric biopsy histopathology.

Results In total, 20 patients were included with a median age of 10.1 [7.6-12.4] years. In keeping with our local practice they had a median of 2 attempts at eradication therapy before being referred for $\mathrm{H}$. pylori culture. On these 20 patients, 15 patients $(75 \%)$ had a confirmed colonisation by $\mathrm{H}$. pylori on histopathology. 14 of these patients (93\%) had successful culture of $\mathrm{H}$. pylori, $1(7 \%)$ had a failed culture.

On these 14 cultures, $1(7 \%)$ had initial growth but failed sensitivities. $3 \mathrm{H}$. pylori cultures (21\% of positive culture) were fully sensitive to amoxicillin, metronidazole and clarithromycin. $4(28 \%)$ were resistant to a single agent $(50 \%$ to metronidazole and 50\% to clarithromycin). 5 (35\%) were resistant to both clarithromycin and another agent $(80 \%$ to metronidazole and 20\% to amoxicillin) and 1 (7\%) was fully resistant to these 3 antibiotics.

After sensitivity-based eradication therapy, 7 patients $(50 \%$ of positive cultures) had ongoing $H$. pylori colonisation (3 confirmed on repeated OGD, 4 confirmed on a positive stool antigen), 4 (28\%) had confirmed eradication (3 confirmed on repeat OGD and 1 on negative stool antigen), and 2 results are still awaited. Out of the 7 patients who failed eradication, $6(85 \%)$ patients had resistant organisms and the remaining patient had poor treatment compliance.

Conclusion Our dedicated H. pylori list has excellent culture recovery (93\%), but its set-up can be challenging.

Our approach to restricting culture to two therapy failures may explain our high rates of targeted antibiotic failure $(50 \%)$, but these patients may also be biased towards therapeutic non-compliance.

Having a better knowledge of the positivity rate of stool antigens in our paediatric population and eradication rate after firstline therapy would be helpful to consciously consider our results.

\section{P27 HYPOPHOSPHATEMIA AFTER TREATMENT OF IRON DEFICIENCY WITH FERRIC CARBOXYLMALTOSE (FCM) INFUSION IN PAEDIATRIC INFLAMMATORY BOWEL DISEASE IN A TERTIARY PAEDIATRIC GASTROENTEROLOGY CENTRE}

Rajkumar Dhandayuthapani, Helen Doble, Fiona Cameron, Manjula Velayudhan Nair, Marcus Auth, Sarang Arun Tamhne, Stephen Allen, Jeng Cheng, Elizabeth Renji. Alder Hey Children's Hospital

10.1136/flgastro-2021-bspghan.37

Background Anaemia is the most common extra intestinal manifestation in paediatric Inflammatory Bowel Disease (pIBD) needing monitoring and supplementation. Intravenous iron is often needed in moderate to severe disease or intolerance to oral iron. Ferric Carboxymaltose (FCM) infusion has been used in our centre. While the risk of hypophosphatemia (HP) associated with FCM is known, (Medicines and Healthcare Products Regulation Agency alert), it is not been quantified in clinical practice.

Aims and Objectives Primary objectives' to report incidence and severity of HP after infusion of FCM in pIBD. Assess time to recovery from $\mathrm{HP}$ with interventions. Secondary objectives' to review patient characteristics and biochemical markers to identify risk factors for FCM infusions associated HP.

Design study We conducted a retrospective electronic records review of pIBD patients who received FCM infusion from November 19-November 20.

Results 24 patients $(13 \mathrm{M})$ received 26 infusions in the period reviewed. The median age was $14.5(12.6-15.9)$. From all the children, $7(29.2 \%)$ were diagnosed with Ulcerative Colitis (UC), 16(66.7\%) with Crohn's Disease and 1(4.2\%) with IBDUnclassified (IBDU). The timing of infusion coincided with: admitted with new diagnosis of pIBD, [10(41.7\%)], flare of disease $[9(37.5 \%)]$ and elective admission [5(20.8\%)] for correction.

Median dose for the 26 infusions was $1000(500-1000) \mathrm{mg}$. Pre-infusion median (IQR) haemoglobin 107.5 (92.7-119.2), MCV 81(74.7-83.2), iron 3.4(2.1-5.5), ferritin 73.1(33.2$115.7)$ and transferrin saturation $4.85(3.6-10.0) \%$. All had normal renal function; median(IQR) urea 2.9(2.32-3.35) and creatinine, 50.5(37-61.7). Pre-transfusion Vitamin D $(n=19)$ median was 38.5(27.5-53.5), with levels being deficient in 13 $(68.3 \%)$ with no association to recovery time.

The biochemical markers are shown in table 1 below, with the change shown as delta change. The delta change was found to be statistically significant for serum phosphate levels. There were no statistically significant association, on univariate analysis, between the delta change in serum phosphate levels and the features of patient demographics or biochemical markers.

All 24 patients had reduction in phosphate level post FCM infusion. In 14/26(53\%), phosphate levels dropped to moderate-severe range; $10 / 14(71.4 \%) \mathrm{HP}$ was moderate $(<0.65$ $\mathrm{mmol} / \mathrm{L}$ ) and in $4 / 14(28.5 \%) \mathrm{HP}$ was severe $(<0.32 \mathrm{mmol} /$ L). The median time of recovery from hypophosphatemia was 14(4-26) days. Those that took longer than 14 days to normalise phosphate $(9,37.5 \%)$ had no features attributable to it.

No serious sequelae of hypophosphatemia were seen. Treatment included a combination of IV phosphate infusion $(6,25 \%)$, oral phosphate supplements $(12,50 \%)$, oral calcium supplements $(15,62.5 \%)$ and vitamin D supplements $(17,70.8 \%)$.

Conclusion HP is frequently seen with FCM infusion. The fall in phosphate post FCM infusion was found to be clinically

\begin{tabular}{lllll} 
Abstract P27 Table 1 \\
\hline Biochemical markers & Baseline & Post-infusion & Delta change & $\mathbf{p}$ \\
\hline Serum phosphate & $1.19 \pm 0.26$ & $0.71 \pm 0.40$ & $-0.48 \pm 0.35$ & $\mathrm{p}<0.0005^{*}$ \\
lonized calcium & $2.35 \pm 0.09$ & $2.37 \pm 0.11$ & $0.02 \pm 0.09$ & $\mathrm{p}<0.284$ \\
Serum potassium & $4.03 \pm 0.52$ & $4.21 \pm 0.37$ & $0.17 \pm 0.62$ & $\mathrm{P}<0.177$ \\
Serum magnesium & $0.82 \pm 0.08$ & $0.77 \pm 0.18$ & $-0.01 \pm 0.07$ & $\mathrm{P}<0.221$ \\
\hline
\end{tabular}


and statistically significant; though none of the patient demographic features or serological markers were found to be associated with the delta change in phosphate to predict high risk patients. The median recovery time of 14 days is less than what is reported in adult reviews. FCM infusions need preassessment, counselling and post infusion monitoring to assess effectiveness and recovery.

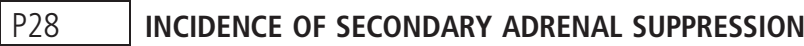 AFTER PROLONGED USE OF GLUCOCORTICOID THERAPY FOR CHILDREN WITH INFLAMMATORY BOWEL DISEASE}

${ }^{1}$ Virginia Chatzidaki, ${ }^{2}$ Rebecca Renji Chungath, 'Sarang Tamhne, 'Marcus Auth, ${ }^{1}$ Fiona Cameron, 'Manjula Velayudhan Nair, ${ }^{1}$ Jeng Haw Cheng, 'Stephen Allen, ${ }^{1}$ Elizabeth Renji, ${ }^{1}$ Jo Blair. ${ }^{1}$ Alder Hey Children's Hospital Trust; ${ }^{2}$ Newcastle medical school

\subsection{6/flgastro-2021-bspghan.38}

Introduction Glucocorticoids (GCs) are used in all forms of paediatric inflammatory bowel disease (IBD) for their antiinflammatory and immunosuppressive effect. Prolonged GC treatment may suppress the hypothalamic-pituitary-adrenal (HPA) axis causing secondary adrenal suppression (SAS), despite using a weaning regime, necessitating the use of hydrocortisone for replacement. The incidence and severity of SAS is poorly predictable and may be related to both the cumulative dose of GCs, but also to individual factors. Standard and low dose short Synacthen tests (SST, LDSST) are used to assess recovery of the HPA axis following a prolonged course of steroids.

Aims To report:

1. the incidence of SAS following GC treatment for IBD in our centre

2. the time to HPA axis recovery in patients with SAS

3. risk factors for SAS

Patients and Methods 33 children with IBD (19M, 10-18 years) previously treated with GCs, who had been investigated for SAS from 01/01/2017 and 30/10/2020 were identified. Baseline information including age, sex, somatometric parameters, IBD diagnosis, age at diagnosis and maintenance treatment were collected. All their GC courses $(n=47)$ in the defined period were reviewed, and those that led to SAS were compared for length and GC doses, previous exposure to GC, tests for HPA axis recovery and the requirement for hydrocortisone replacement therapy.

Results Of the 33 children, $15 / 33(45.5 \%)$ had ulcerative colitis (UC), 10/33 (30.3\%) had Crohn's Disease (CD) and 8/33 (24.2\%) were unclassified (IBDU). 10 children that were tested using non-standard tests for adrenal suppression were excluded.

23 children had their HPA axis recovery tested after 24 GC courses with LDSST $(19 / 24,79.2 \%)$ or SST $(5 / 24$, 20.8\%). 16/23 children had steroid courses of $\geq 12$ weeks, and $7 / 23$ had a shorter course ( $<11$ weeks) but had extended courses in the past (4/7) and/or high dose IV steroid (3/7). $14 / 23(60.9 \%)$ children had suboptimal response with peak cortisol range $129-441 \mathrm{nmol} / \mathrm{L}$ (table 1). 8/14 retests were available in the study period, 5/8(62.5\%) children recovered HPA axis function in a mean period of $6.83 \pm$ SD2.4 months and $3 / 8$ remained suppressed after a mean period of 4.6 \pm SD2.7 months.
Abstract P28 Table 1 Patients with inflammatory bowel disease tested with SSTs

\begin{tabular}{|c|c|c|c|}
\hline Characteristics & $\begin{array}{l}\text { Adrenal } \\
\text { Insufficiency } \\
(n=14)\end{array}$ & $\begin{array}{l}\text { Normal } \\
\text { Response } \\
(n=9)\end{array}$ & $\begin{array}{l}\mathrm{p}- \\
\text { value }\end{array}$ \\
\hline Age of Diagnosis, mean (range in yrs) & $11.7(6-15)$ & $11.6(9-15)$ & 0.29 \\
\hline \multicolumn{4}{|l|}{ Gender, n (\%) } \\
\hline - Male & $7(50 \%)$ & $5(55.6 \%)$ & 0.56 \\
\hline - Female & $7(50 \%)$ & $4(44.4 \%)$ & \\
\hline \multicolumn{4}{|l|}{ IBD phenotype, n(\%) } \\
\hline - Crohn's Disease & $4(28.6 \%)$ & $2(22.2 \%)$ & 0.56 \\
\hline - Ulcerative Colitis & $8(57.1 \%)$ & $5(55.6 \%)$ & 0.63 \\
\hline - IBD unclassified & $2(14.3 \%)$ & $2(22.2 \%)$ & 0.52 \\
\hline \multicolumn{4}{|l|}{ Other non-systemic GCs } \\
\hline - Rectal GCs & $5(35.7 \%)$ & $4(44.4 \%)$ & 0.50 \\
\hline - Inhaled CS (fluticasone) & 0 & $1(11.1 \%)$ & \\
\hline Total GCs courses, mean (range) & $1.79(1-3)$ & $2.33(1-4)$ & 0.10 \\
\hline \multicolumn{4}{|l|}{ Indication for test n (\%) } \\
\hline - $\mathrm{GCs}$ course $\geq 12$ weeks & $11(78.6 \%)$ & $6(66.7 \%)$ & 0.43 \\
\hline - Previous GCs course $\geq 12$ weeks & $5(35.7 \%)$ & $7(77.8 \%)$ & 0.06 \\
\hline $\begin{array}{l}\text { - Use of } 10-20 \mathrm{mg} / \mathrm{kg} \text { (high dose) IV } \\
\text { Methylprednisolone }\end{array}$ & $5(35.7 \%)$ & 0 & \\
\hline Serum Basal Cortisol, mean (range) (nmol/L) & $177.6(<50-364)$ & $246.6(131-406)$ & 0.05 \\
\hline
\end{tabular}

The mean duration of courses that induced SAS was 154 \pm 86.8 days compared to $131.6 \pm 63$ days in those without SAS, $\mathrm{t}=0.44, \mathrm{p}=0.33$, and the previous days of exposure were 219 \pm 95 days for those with SAS compared 316.5 \pm 154.2 days for those without SAS, $\mathrm{t}=-1.2, \mathrm{p}=0.13$.

High dose IV methylprednisolone (10-20 mg/kg) was used in $5 / 24(20.8 \%)$ courses with $5 / 5(100 \%)$ and $3 / 5$ (60\%) inducing HPA axis suppression on the first testing and retesting respectively. Standard dose $(1.6 \mathrm{mg} / \mathrm{kg})$ IV methylprednisolone followed by oral weaning GC course was used in 9/24 (37.5\%) courses and 5/9 (55.6\%) induced HPA axis suppression. Standard dose oral prednisolone $(1-2 \mathrm{mg} / \mathrm{kg}$ ) was used in $10 /$ $24(41.7 \%)$ of the courses and 5/10 (50\%) induced SAS.

Conclusion SAS was detected in $60.9 \%$ of our IBD patients who were tested after GCs therapy. Our data support previous reports that additional factors to the duration of treatment with GCs, as the use of high dose of intravenous GCs, may influence the risk of SAS.

\section{P29 INDICATIONS OF PAEDIATRIC ENDOSCOPIES AND CORRELATION BETWEEN RESULTS AND CLINICAL OUTCOMES}

${ }^{1}$ Mara Popescu, ${ }^{1}$ Badra Farah, ${ }^{2}$ Mohamed Mutalib. 'King's College London; ${ }^{2}$ Evelina Children's Hospital, London

\subsection{6/flgastro-2021-bspghan.39}

Background Paediatric endoscopies are expensive and invasive procedures requiring general anaesthetic and should be performed to answer specific clinical questions. There is an increase in number of endoscopies performed in children but no epidemiological evidence of increasing paediatric GI disease burdens. Biopsies are also routinely performed during endoscopies adding to overall procedure cost.

Aims This study aimed to assess the indications of paediatric endoscopy, the association between endoscopic and histological 